\title{
HRE1 and HRE2, two hypoxia-inducible ethylene response factors, affect anaerobic responses in Arabidopsis thaliana
}

\author{
Francesco Licausi ${ }^{1,2}$, Joost T. van Dongen ${ }^{2}$, Beatrice Giuntoli ${ }^{3}$, Giacomo Novi ${ }^{1}$, Antonietta Santaniello ${ }^{1}$, Peter Geigenberger ${ }^{2,4}$ \\ and Pierdomenico Perata, ${ }^{1, *}$ \\ ${ }^{1}$ Plant Lab, Scuola Superiore Sant'Anna, Piazza Martiri della Libertà 33, 56127 Pisa, Italy, \\ ${ }^{2}$ Max Planck Institute of Molecular Plant Physiology, Am Muehlenberg 1, 14476 Golm, Germany, \\ ${ }^{3}$ Department of Crop Plant Biology, University of Pisa, Via Mariscoglio 34, 56124 Pisa, Italy, and \\ ${ }^{4}$ Department Biologie I, Ludwig-Maximilians-Universität München, 82152 Martinsried, Germany
}

Received 3 November 2009; revised 13 January 2010; accepted 14 January 2010; published online 25 February 2010.

*For correspondence (fax +39 050 2216532; e-mail p.perata@sssup.it).

\begin{abstract}
SUMMARY
Plants often experience challenging hypoxic conditions imposed by soil waterlogging or complete flooding. In rice, Sub1A, a flooding-induced ethylene responsive factor (ERF) plays a crucial role in submergence tolerance. In this study, we examined two Arabidopsis Hypoxia Responsive ERF genes (HRE1 and HRE2), belonging to the same ERF group as Sub1A. Transgenic Arabidopsis plants, which over-expressed HRE1, showed an improved tolerance of anoxia, whereas a double-knockout mutant hre1hre2 was more susceptible than the wild type. HRE1 over-expressing plants showed an increased activity in the fermentative enzymes pyruvate decarboxylase and alcohol dehydrogenase together with increased ethanol production under hypoxia, but not in normoxia. Whole-genome microarray analyses suggested that an over-expression of HRE1, but not HRE2, increased the induction of most anaerobic genes under hypoxia. Real-time quantitative (q)PCR analyses confirmed a positive effect of HRE1 over-expression on several anaerobic genes, whereas the double-knockout mutant hre1hre2 showed a decreased expression in the same genes after $4 \mathrm{~h}$ of hypoxia. Single-knockout mutants did not show significant differences from the wild type. We found that the regulation of HRE1 and HRE2 by low oxygen relies on different mechanisms, since HRE1 requires protein synthesis to be induced while HRE2 does not. HRE2 is likely to be regulated post-transcriptionally by mRNA stabilization. We propose that HRE1 and HRE2 play a partially redundant role in low oxygen signalling in Arabidopsis thaliana, thus improving the tolerance of the plant to the stress by enhancing anaerobic gene expression and ethanolic fermentation.
\end{abstract}

Keywords: hypoxia, anoxia, ERF, ADH, transcription factor, Arabidopsis.

\section{INTRODUCTION}

Plants often have to cope with oxygen shortages during their lifetime. In fact, even with normal oxygen concentrations in the atmosphere, plant tissues can experience hypoxia because of impediments to intracellular oxygen transport (Geigenberger, 2003; van Dongen et al., 2004). Moreover, hypoxia can occur when external oxygen availability decreases if the whole plant or non-photosynthetic organs become flooded, since gas diffusion is four-orders of magnitude slower in water than in a gaseous atmosphere (Armstrong, 1979).

Lack of oxygen causes a reduction in respiratory efficiency and, as a consequence, in energy production (Gupta et al., 2009). Therefore, under anoxia ATP synthesis is mostly provided by glycolysis coupled with NAD regenerative pathways, including ethanolic fermentation and alanine production (Ismond et al., 2003; Ricoult et al., 2006). Additionally, in rice (Oryza sativa), nitrite can substitute for $\mathrm{O}_{2}$ as the final electron acceptor in the mitochondrial electron transport chain (Stoimenova et al., 2007).

Both the transcriptional and translational regulations of specific genes play a crucial role in the adaptation of plants to oxygen limitation (Licausi and Perata, 2009). However, despite several previous studies that have carried out detailed overviews of the global transcriptome and proteome, only a few transcription factors regulating these events have been described so far.

Analyses of the promoter sequences of hypoxia-induced genes, such as alcohol dehydrogenase $(A D H)$, pyruvate Journal compilation (๑) 2010 Blackwell Publishing Ltd 
decarboxylase (PDC) and sucrose synthase (SUSY) in several plant species have contributed to the identification of several DNA elements putatively responsible for hypoxia inducibility (Dolferus et al., 1994; Mohanty et al., 2005). Transcription factors belonging to the MYC, MYB, AP2/ERF and NAC families have been reported to be able to up-regulate $A D H$ expression in Arabidopsis (Abe et al., 2003; Papdi et al., 2008; Christianson et al., 2009). However, only MYB2 has been directly related to hypoxic stress (Hoeren et al., 1998) since it binds the GT motif, a DNA element that is necessary for induction of $A D H$ under hypoxic conditions. More recently, Sub1A, a transcription factor belonging to the VII group of the ethylene responsive factor (ERF) family (Nakano et al., 2006), has been demonstrated to play a major role in the submergence tolerance of lowland rice varieties (Fukao et al., 2006; Xu et al., 2006). According to the model proposed, the induction of Sub1A reduces gibberellin (GA) responsiveness, thus leading to shoot elongation, by means of a process involving ethylene (which induces Sub1A) and an increase in the level of GA signalling repressors such as SLR1 and SLRL1 (Fukao and Bailey-Serres, 2008). In this way, Sub1A promotes a 'quiescent strategy' in order to avoid any unnecessary energy consumption caused by hormone-mediated elongation in the submerged tissues (Perata and Voesenek, 2007). In the opposite fashion, the ERF transcription factors SNORKEL1 and SNORKEL2 promote GA-mediated internode elongation in deep-water rice varieties (Hattori et al., 2009), promoting an 'escape strategy' to survive flooding (Voesenek and Bailey-Serres, 2009).

Apart from Sub1A, which is not conserved in all rice varieties, other members of the ERF group VII have been reported to be up-regulated under low-oxygen conditions in Arabidopsis, rice and poplar (Populus trichocarpa) (Loreti et al., 2005; Lasanthi-Kudahettige et al., 2007; Kreuzwieser et al., 2009). These data suggest that this family of transcription factors is a strongly conserved element in the regulation of hypoxic responses in higher plants.

The ERF transcription factors are so called because the first members of this family to be studied showed ethylene responsiveness (Ohme-Takagi and Shinshi, 1995). However, further characterization of the whole family clearly demonstrated that each ERF is regulated in a specific manner and, therefore, this acronym is nowadays associated with a conserved domain of 60-70 amino acids responsible for DNA binding (Sakuma et al., 2002; Nakano et al., 2006).

Transcriptional regulators belonging to the AP2/ERF family play a role in many processes, encompassing development (Elliott et al., 1996; Boutilier et al., 2002) and responses to both biotic (Yamamoto et al., 1999) and abiotic stresses (Stockinger et al., 1997; Liu et al., 1998; Xu et al., 2006). Several members of the ERF family from different plant species have been demonstrated to regulate genes involved in scavenging reactive oxygen species (ROS) (Ogawa et al., 2005; Wu et al., 2008). When over-expressed, many of these members are also able to increase tolerance to different stress conditions (Weiste et al., 2007; Wu et al., 2008; Youm et al., 2008).

A DNA element named the GCC box (AGCCGCC) has been shown to be recognized by most members of the AP2/ERF family (Ohme-Takagi and Shinshi, 1995), even though a number of other motifs have been recently demonstrated to be specifically bound by different ERF transcription factors, despite the high conservation of the amino acidic sequence of the DNA-binding domain (Sasaki et al., 2007; Welsch et al., 2007; Maeo et al., 2009).

This conservation among species and the extent of the hypoxic induction of some group VII ERF genes point to their involvement in low-oxygen signalling in plants. In this paper, we describe two group VII ERFs and highlight their role in hypoxic responses in Arabidopsis thaliana.

\section{RESULTS}

\section{HRE1 and HRE2 encode two nuclear-localized ERFs specifically up-regulated under low oxygen in Arabidopsis}

There are five group VII ERF genes in the Arabidopsis genome, namely At1g53910 (RAP2.12), At1g72360, At2g47520, At3g14230 (RAP2.2) and At3g16770 (RAP2.3 also known as AtEREBP). These ERFs are characterized by a conserved DNA-binding domain, by the extremely conserved N-terminal motif MCGGAV/IISD and by the leucine/isoleucine and tryptophan residues at the C-terminus (Figure 1a). In order to estimate the evolutionary relatedness among the members of this group, we used a neighbour joining method to build a phylogenetic tree of the ERF VII proteins from Arabidopsis, rice, maize (Zea mays), grapevine (Vitis vinifera) and poplar (Figure 1b). A separation between dicot and monocot genomes appeared evident. At1g72360, At3g13230 and At1g53910 cluster together with at least another group VII ERF belonging to the dicot genomes considered. At2g47520 and At3g16770 also cluster together with sequences from grapevine and poplar, although in different groups. The rice Sub1 and Snorkel proteins, which regulate the submergence response, cluster together in a rice-specific group.

We carried out a detailed analysis of the expression of the five Arabidopsis members of ERF group VII under hypoxia (Figure 2a). The expression of RAP2.3 was unaffected by hypoxia, while the mRNA level of RAP2.2 and RAP2.12 declined following the low-oxygen treatment (Figure 2a). At1g72360 (AtERF\#73, according to Nakano et al., 2006) and At2g47520 (AtERF\#71) were induced by low oxygen and were therefore named HRE1 and HRE2 (Hypoxia Responsive ERFs; Figure 2a). The HRE1 expression pattern under hypoxia highlighted a peak of expression $2 \mathrm{~h}$ after the onset of hypoxia (Figure 2a). HRE2 mRNA was barely detectable in 
(a)
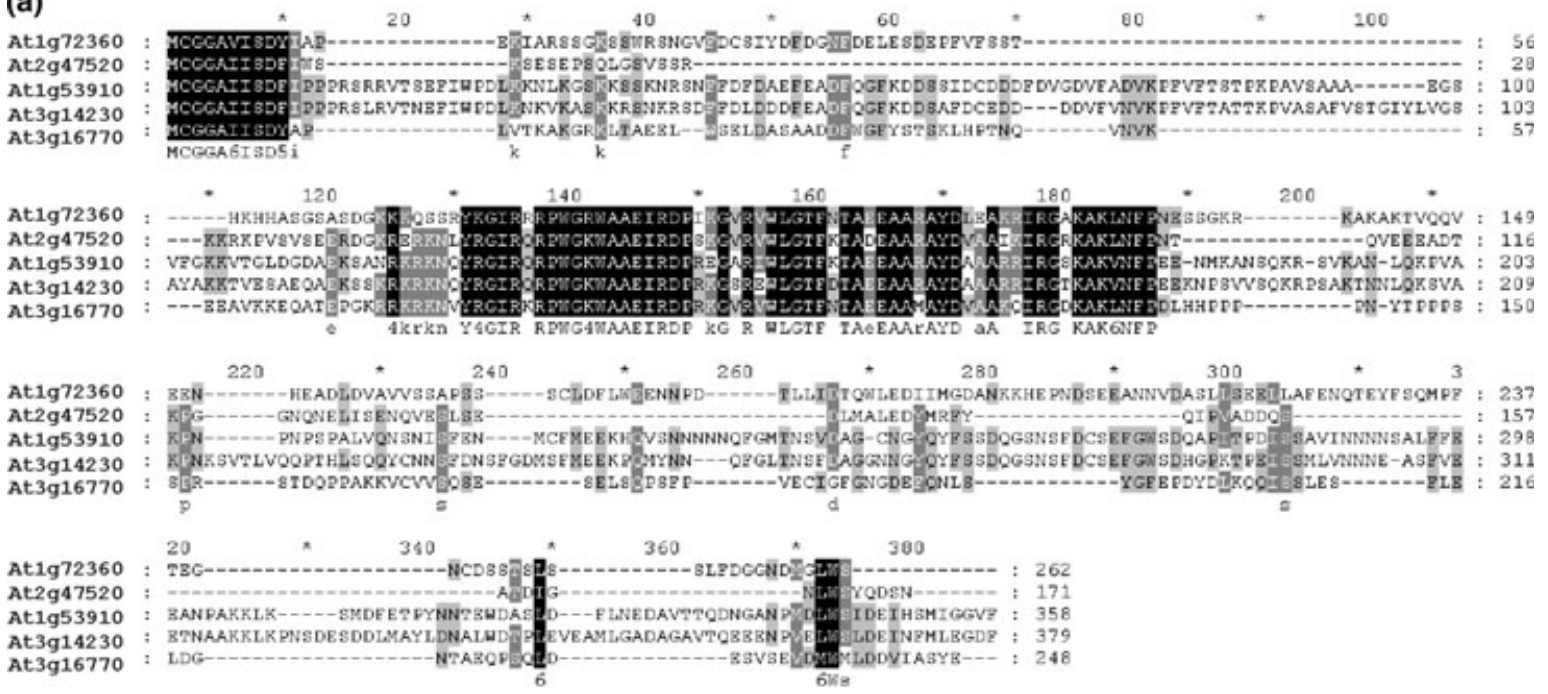

(b)

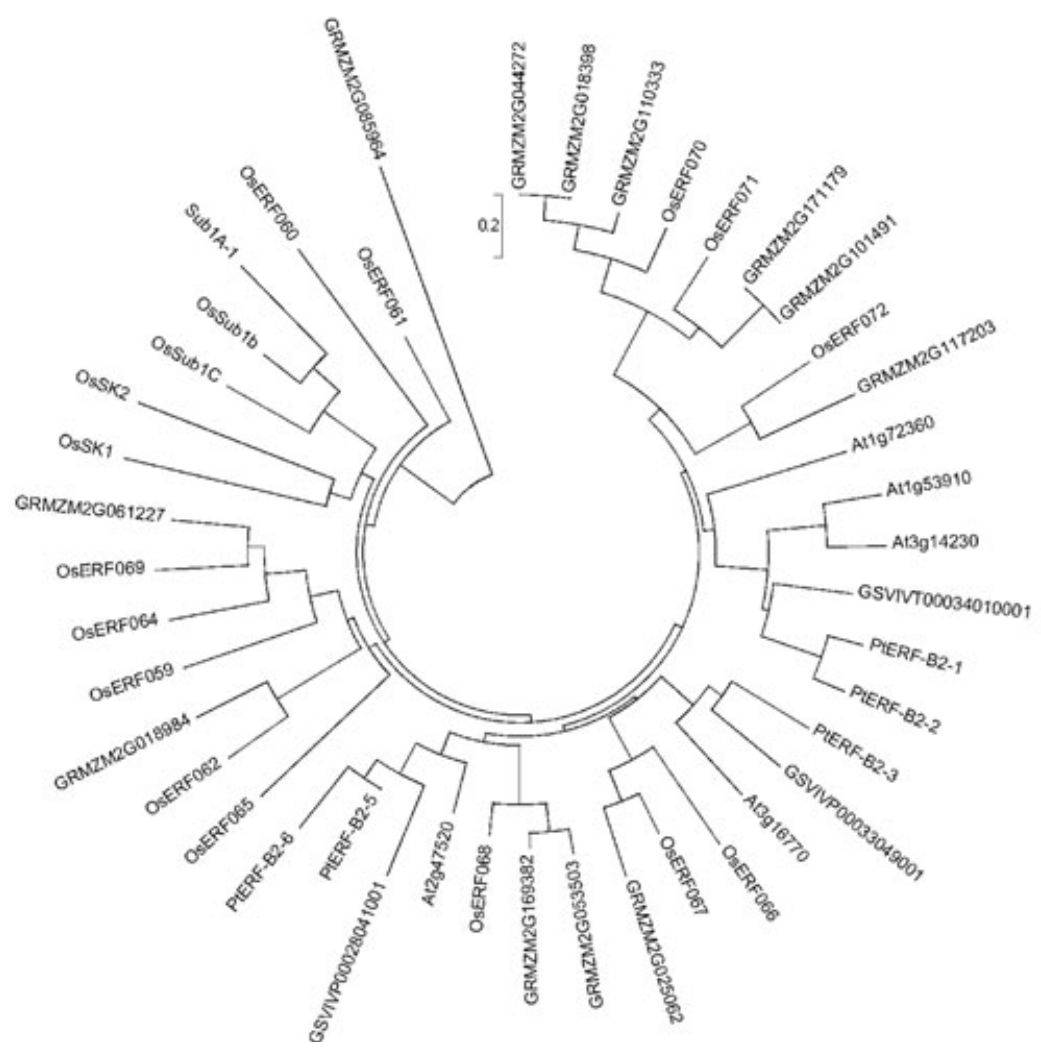

Figure 1. The group VII ethylene responsive factors (ERFs) in Arabidopsis.

(a) Alignment of the protein sequences of the Arabidopsis group VII ERFs. Amino acidic sequences were aligned using ClustalW2 software (http://www.ebi.ac.uk/ Tools/clustalw2/index.html) and Genedoq (Nicholas et al., 1997) was used to analyse/display the alignment. Shaded boxes represent different levels of conservation among the ERF VII proteins in Arabidopsis (black $=100 \%$, grey $=80 \%$, light grey $=60 \%$ ).

(b) Phylogenetic tree illustrating the relatedness of group VII ERFs among plant species whose genome has been sequenced. An unrooted neighbour-joining tree was created using MEGA4 (Tamura et al., 2007). The distance bar is shown on the top of the tree. The protein sequences used were: Arabidopsis thaliana (At1g53910 At1g72360, At2g47520, At3g14230, At3g 16770), Populus trichocarpa (PtERF-B2-1, PtERF-B2-2, PtERF-B2-3, PtERF-B2-5, PtERF-B2-6) as named according to Zhuang et al. (2008), Vitis vinifera (GSVIVP00028041001, GSVIVT00034010001, GSVIVP00033049001), Oryza sativa (OsERF059, OsERF060, OsERF061, OsERF062, OsERF064, OsERF065, OsERF066, OsERF067, OsERF068, OsERF069, OsERF070, OsERF071, OsERF072 as named according to Nakano et al., 2006; OsSub1A, OsSub1B, OsSub1C as named according to Fukao et al., 2009; OsSK1 and OsSK2 from cultivar Bhadua as named according to Hattori et al., 2009) and Zea mays (GRMZM2G053503, GRMZM2G025062，GRMZM2G171179，GRMZM2G101491，GRMZM2G110333，GRMZM2G117203，GRMZM2G169382，GRMZM2G061227，GRMZM2G018984، GRMZM2G044272, GRMZM2G018398, GRMZM2G018398, GRMZM2G085964). 
(a)
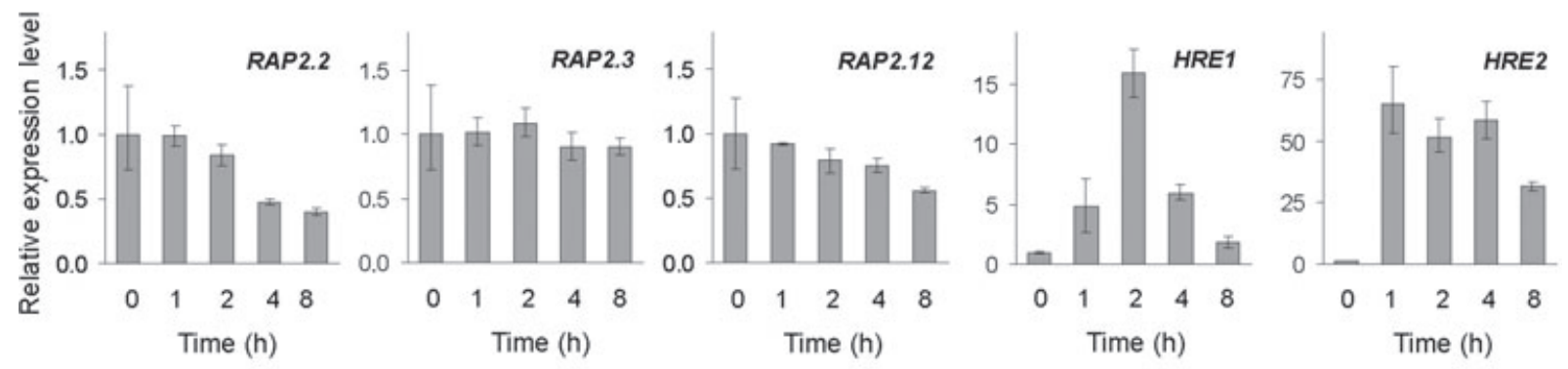

(b)

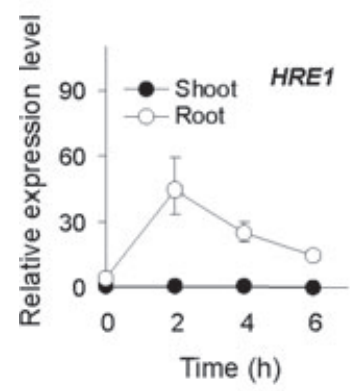

(c)

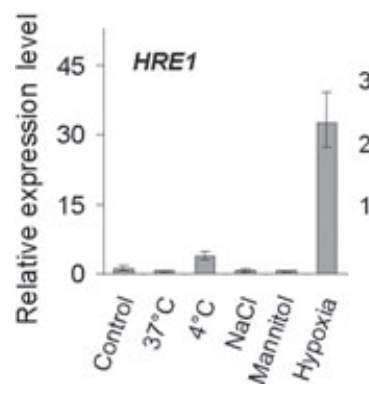

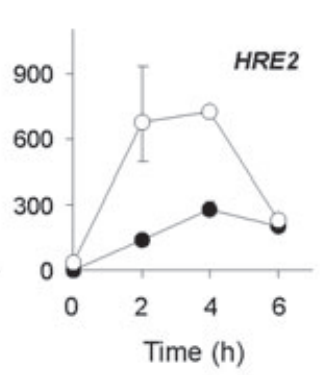

(d)
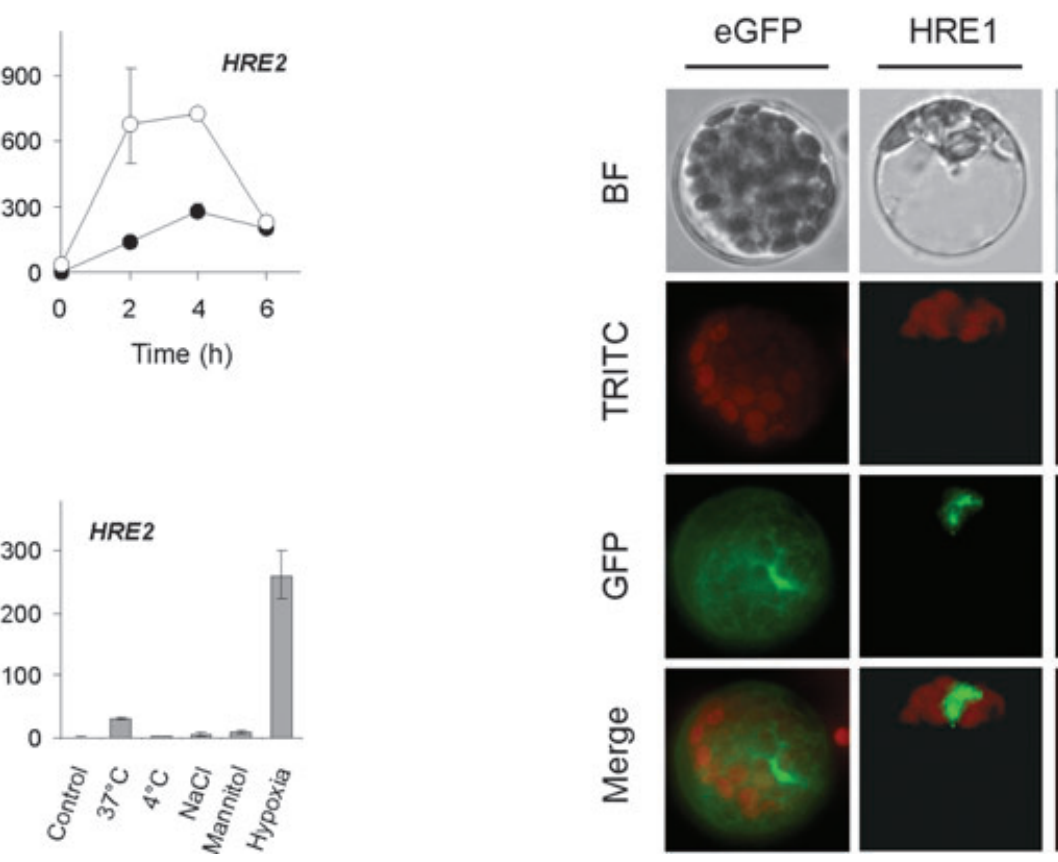
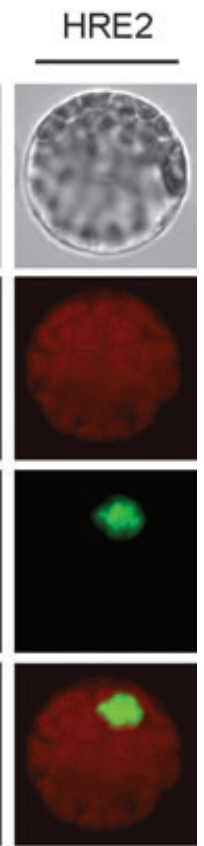

Figure 2. Expression profile of Arabidopsis group VII ethylene responsive factor (ERF) genes.

(a) Effects of hypoxia on the expression of group VII ERFs. Seven-day-old Arabidopsis seedlings were treated under hypoxia ( $\left.1 \% \mathrm{O}_{2}\right)$. Relative expression levels of RAP2.2 (At3g14230), RAP2.3 (At3g16770), RAP2.12 (At1g53910), HRE1 (At1g72360) and HRE2 (At2g47520) were measured. Relative expression levels are shown as fold change values $(1=$ time 0$)$. Data are mean $\pm \operatorname{SD}(n=3)$.

(b) Pattern of expression of HRE1 and HRE2 under hypoxia. Fifteen-day-old seedlings were treated under hypoxia ( $1 \% \mathrm{O}_{2}$ ). The mRNA level in shoots (filled circles) and roots (empty circles) was measured by real-time quantitative (q)PCR. Relative expression levels are shown as fold change values $(1=$ shoot, time 0$)$. Data are mean $\pm \mathrm{SD}(n=3)$.

(c) Relative expression levels of HRE1 and HRE2 in response to different abiotic stresses. Fifteen-day-old seedlings were treated under various abiotic stress conditions for $3 \mathrm{~h}$. The mRNA level in whole seedlings was measured by real-time qPCR. Relative expression levels are shown as fold change values ( $1=$ control). Data are mean $\pm \mathrm{SD}(n=3)$.

(d) Subcellular localization of HRE1 and HRE2 proteins in transiently transformed Arabidopsis mesophyll protoplasts. Pictures were taken using bright-field (BF), chlorophyll (TRITC) and GFP (GFP) filters.

normal air (data not shown) but its mRNA abundance increased rapidly under hypoxia (Figure 2a). Previous microarray analyses reported high HRE2 expression under prolonged ( $48 \mathrm{~h}$ ) hypoxia ( 1 and $4 \%$ oxygen) but its expression declined when the oxygen availability was higher than $4 \%$ (van Dongen et al., 2009).

In general, most of the typical anaerobic genes are either predominantly or exclusively expressed in the roots, where hypoxia is experienced more frequently. Indeed, both HRE1 and HRE2 were mostly expressed in the hypoxic root (Figure 2b). HRE1 is not expressed in shoots, even under hypoxia, while HRE2 is up-regulated ubiquitously, even though the expression level in the root is higher than in the shoot (Figure 2b).

The most obvious effect of an oxygen shortage is an energy deficit. However, energy deprivation is a common consequence of almost all abiotic stresses that negatively affect photosynthesis and/or respiration (Baena-González 
and Sheen, 2008). To verify the hypoxia specificity of the induction of both HRE1 and HRE2, we analysed the effects of a set of abiotic stresses on Arabidopsis seedlings by means of qPCR. The results showed that hypoxia was the strongest trigger for the expression of both HRE1 and HRE2 (Figure $2 \mathrm{c}$ ). In addition to hypoxia, cold and heat also had a modest effect on the expression of HRE1 and HRE2, respectively (Figure 2c).

Some group VII members of the ERF family have been reported to function as positive regulators of transcription, and RAP2.3 has been shown to be localized in the nucleus (Pan et al., 2001). Transient transformation of mesophyll protoplasts with a construct bearing a GFP-tagged version of HRE1 and HRE2 showed that both gene products are indeed localized in the nucleus (Figure $2 \mathrm{~d}$ ).

\section{HRE1 and HRE2 contribute to tolerance of anoxia in Arabidopsis seedlings}

In rice, Sub1A, a group VII ERF, plays a crucial role in submergence tolerance (Xu et al., 2006). Both HRE1 and HRE2 may play a similar role in Arabidopsis.

We decided to investigate the low-oxygen tolerance of Arabidopsis lines with altered expression of HRE1 and HRE2. We therefore produced Arabidopsis plants expressing HRE1 and HRE2 under control of the constitutive 35S CaMV promoter. We also obtained homozygous T-DNA insertion Salk lines from the European Arabidopsis Stock Center (NASC). A mutant bearing an insertion in the $5^{\prime}$ untranslated region (UTR) of HRE1 was selected (Salk_023445) while for HRE2 a mutant characterized by T-DNA insertion in the middle of the second exon was available (Salk_052858). Real-time qPCR analysis of HRE expression confirmed that production of the mature mRNA coding for the full-length protein was abolished in all the Salk lines investigated (Figure S1a in Supporting Information). hre1 and hre2 lines were crossed to generate a double homozygous knockout mutant (hre1hre2). Neither the over-expressers nor the mutant lines obtained showed an evident phenotype at any developmental stage examined (see Figure S1b for the rosette stage).

Tolerance tests using hypoxia showed that Arabidopsis can easily survive several days in a $1 \% \mathrm{O}_{2}$ atmosphere in the dark (not shown). Moreover, the phenotypes observed after this period resembled those of prolonged darkness and carbon starvation stress, which are distinct from oxygen deficiency stress. We therefore decided to expose Arabidopsis HRE over-expressers and mutants to a few hours' anoxia to compare their survival. Wild-type plants (Columbia-0, Col-0) showed a 70\% survival after $8 \mathrm{~h}$ under anoxia, but their tolerance dropped to about $10 \%$ after $10 \mathrm{~h}$ (Figure $3 a, b$ ). The tolerance of the individual mutants (hre1 and $h r e 2)$ to anoxia was comparable to the wild type, while the hre1hre2 mutant showed a significantly lower tolerance after $8 \mathrm{~h}$ of anoxia. Conversely, the 35S::HRE1, but not the
35S::HRE2, plants showed enhanced anoxia tolerance, with $92 \%$ surviving after $8 \mathrm{~h}$ of anoxia and $67 \%$ after $10 \mathrm{~h}$ (Figure 3a,b).

A detailed anoxic time-course confirmed the lower tolerance displayed by the hre1hre2 genotype (Figure 3c,d). Enhancement of tolerance to anaerobiosis in plants often relies, at least partly, on the ability to regenerate $\mathrm{NAD}^{+}$ through ethanol fermentation (Bailey-Serres and Voesenek, 2008). The increased tolerance in 35S::HRE1 plants could be due to an enhancement of the alcoholic fermentative pathway. Indeed, the 35S::HRE1 roots showed ADH and PDC activities that were significantly higher than the wild type (Col-0) (Figure 4a). Interestingly, activities of both ADH and PDC in 35S::HRE1 plants were already higher than in the wild type in aerobic conditions. To verify whether the increased PDC and ADH activities correlated with enhanced fermentation in vivo, we measured ethanol production. 35S::HRE1 plants showed an increased hypoxic ethanol production, whereas the double mutant displayed a lower ethanol accumulation (Figure 4a). Under aerobic conditions, no significant difference was found among the genotypes (Figure 4a). Additionally, we analysed the time-course of ethanol production in the wild type, double mutant and 35S::HRE1 seedlings (Figure 4b). The results indicated that the hre1hre2 mutant produced less ethanol than the wild type at all time points, while 35S::HRE1 seedlings showed increased ethanol production after 12 and $24 \mathrm{~h}$ under hypoxia (Figure 4b).

\section{HRE1 and HRE2 affect the expression of anaerobic genes under low oxygen}

The phenotypic and biochemical changes that we observed in $35 S$ ::HRE 1 plants suggested that HRE1 is involved in the transcriptional regulation of anaerobic genes, thus playing a role in mechanisms of adaptation to low oxygen. A microarray analysis was carried out on the 35S::HRE1 and 35S::HRE2 lines compared with the wild type (Col-0) under aerobic and hypoxic conditions $\left(4 \mathrm{~h}\right.$ at $\left.1 \% \mathrm{O}_{2}\right)$. In air, $H R E 1$ over-expression significantly influenced (adjusted $P$-value $\leq 0.05)$ five genes in addition to HRE1 itself (Table S1) Interestingly, $A D H$ was among the up-regulated genes (Table S1). Under hypoxia, 22 genes were significantly up-regulated (twofold or more) and 15 were down-regulated (twofold or less) in 35S::HRE1 seedlings compared with the wild type (Table S2). Among the up-regulated genes, At1g55810 (putative uracil phosphoribosyltransferase) At4g30380 (expansin-like B2 precursor), At5g54740 (lipid transfer family protein), At4g32840 (putative phosphofructokinase), At1g77200 (AP2 transcription factor TINY) and At2g22880 (VQ domain-containing protein) have also been previously reported to be induced by low oxygen in other microarray analyses (Branco-Price et al., 2005, 2008; Loreti et al., 2005). Among the 15 genes significantly downregulated, At5g24210, encoding for a lipase class 3 protein, 
(a)
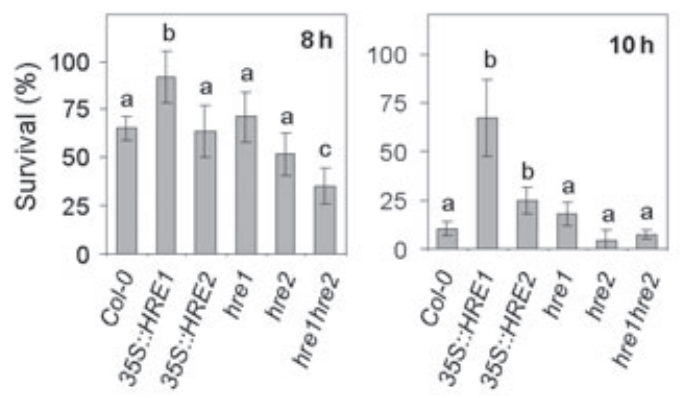

(c)

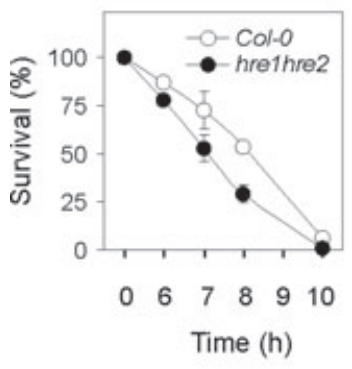

(d)

Control (b)
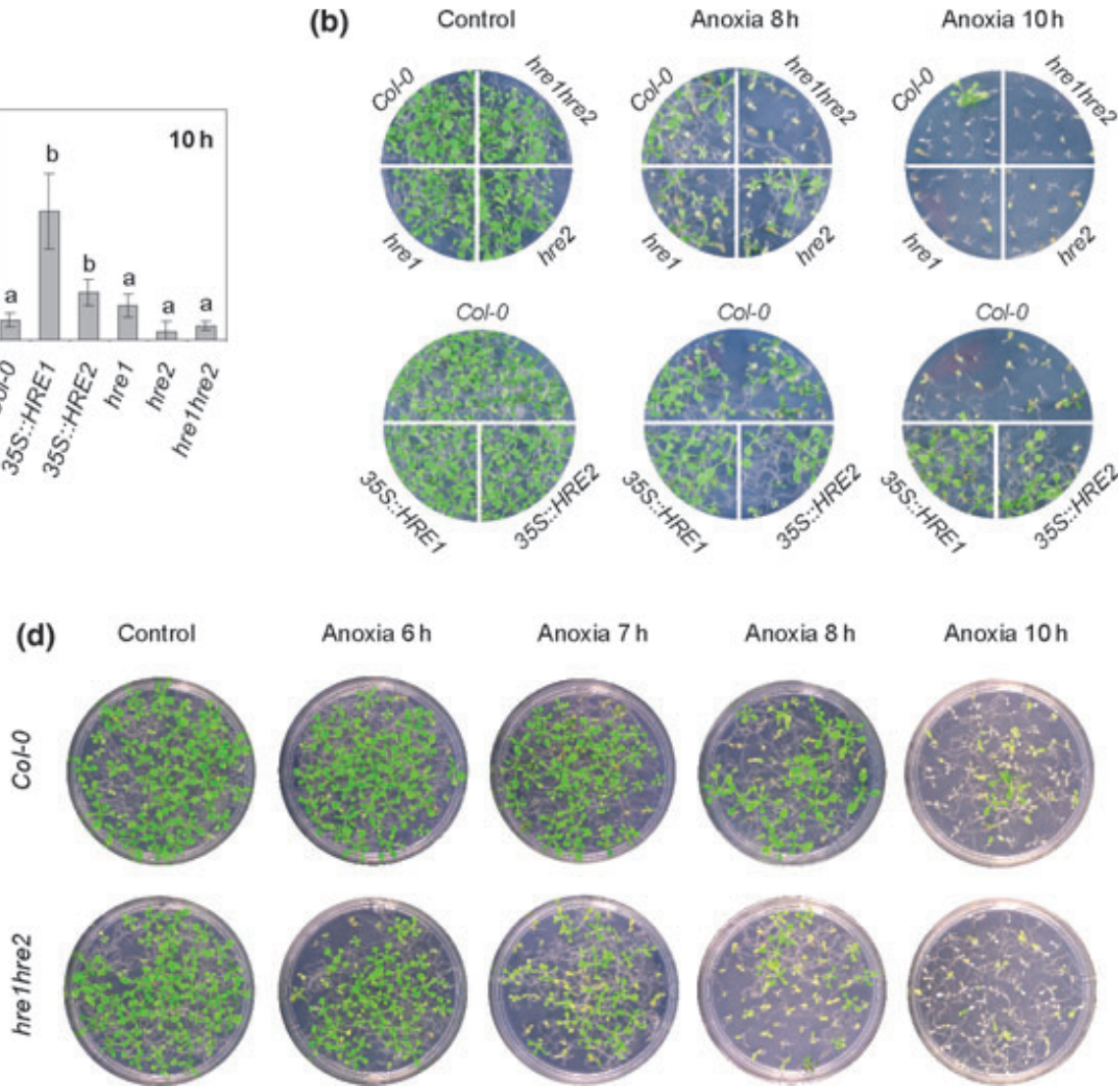

Anoxia $10 \mathrm{~h}$
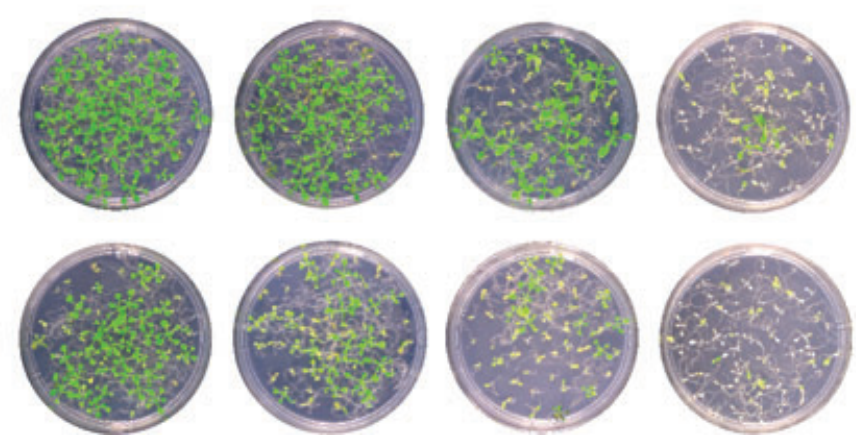

Figure 3. Effects of HRE1 and HRE2 on tolerance of anoxia.

(a) Survival rates of 7-day-old Arabidopsis plants treated under anoxia for 8 and $10 \mathrm{~h}$. Fifteen seeds were sown in each plate sector. After the anoxic treatment, plants were transferred to air and surviving plants were counted after a 10-day recovery period. Survival rates were calculated as a percentage. Data are mean survival rates $\pm \mathrm{SD}$ ( $n=5$ plates containing at least 15 seedlings each).

(b) Phenotypes of the wild-type, mutant and over-expresser plants after a 14-day recovery after the anoxic treatments.

(c) Survival rates of 7-day-old Arabidopsis plants treated under anoxia for 6, 7, 8 and 10 h. Fifty seeds were sown in each plate ( $n=4$ for each time point). After the anoxic treatment, plants were transferred to air and surviving plants were counted after a 10-day recovery period. Survival rates were calculated as a percentage. Data are mean survival rates $\pm \mathrm{SD}$ ( $n=4$ plates containing at least 50 seedlings each).

(d) Phenotypes of the wild type (Col-0) and the double mutant hre1hre2 after a 14-day recovery after the anoxic treatments.

At3g04290, called ATLTL1, a lipase involved in cadmium tolerance, At2g47000, a P-glycoprotein (PGP4), At5g45950, a GDSL-lipase, and At2g04040 (ATDTX1) are involved in lipid metabolism and membrane remodelling related to stress tolerance. In addition ELIP2, PLA2A and CSD2, encoding enzymes involved in scavenging and protection against ROS production, are less expressed in the 35S:HRE1 seedlings.

In aerobic conditions, the over-expression of HRE2 significantly affects the expression of 31 genes, among which 26 are down-regulated (Table S3). Surprisingly, included in this list are $A D H$, non-symbiotic haemoglobin (HB1), $P D C$, At5g66985, At4g33560 and At3g48100 that have been shown to be up-regulated by low oxygen in several experiments (Branco-Price et al., 2005; Loreti et al., 2005; van Dongen et al., 2009). However, the hypothesis that HRE2 acts as a negative regulator of anaerobic gene expression cannot be accepted. In fact, under hypoxic conditions, HRE2 overexpression does not affect the expression of typical anaer- obic genes (Table S4). To gain a further overview of anaerobic genes, we selected those genes whose expression was increased by hypoxia more than eightfold in the wild type. This group was then compared with previous microarray analyses on the anaerobic transcriptome in similar conditions (Branco-Price et al., 2005, 2008; Loreti et al., 2005; van Dongen et al., 2009). All 48 genes were induced by lowoxygen treatments and were therefore designated as an anaerobic cluster (Table S5). A two-way ANOvA analysis of the anaerobic cluster showed a significant increase in gene expression in 35S::HRE1 compared with the wild-type under hypoxia, but not in 35S::HRE2 (Figure 5). We investigated the possible effects of HRE1 and HRE2 on the expression of anaerobic genes under hypoxia by analysing a detailed timecourse using a more sensitive real-time qPCR procedure. We selected ADH, HB1, SUCROSE SYNTHASE1 (SUS1) and SUCROSE SYNTHASE4 (SUS4) as good markers of the anaerobic response and tested their expression at different 


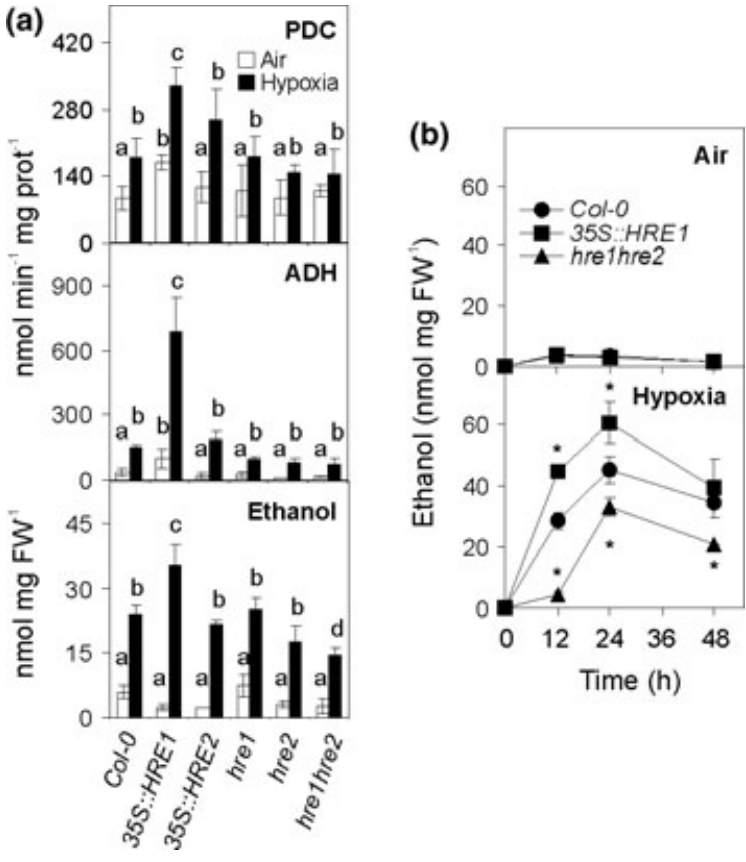

Figure 4. Effects of HRE1 and HRE2 on ethanolic fermentation.

(a) Fermentative enzyme activities [pyruvate decarboxylase (PDC) and alcohol dehydrogenase $(A D H)]$ and ethanol accumulation in roots from 15-day-old plants and in 7-day-old seedlings, respectively, treated for $12 \mathrm{~h}$ with air (empty bars) and $1 \% \mathrm{O}_{2}$ (filled bars) in the dark. The results shown are mean $\pm \mathrm{SD}$ $(n=3)$. A two-way ANOva ( $P$-value $\leq 0.01)$ pairwise comparison was used to assess the significance of the differences found between the 'HRE' genotypes and the wild type (Col-0) under aerobic and hypoxic conditions.

(b) Ethanol production over $48 \mathrm{~h}$ in 7-day-old seedlings treated with air and $1 \%$ $\mathrm{O}_{2}$ in the dark. The results shown are mean $\pm \mathrm{SD}(n=3)$. A two-way ANOvA $(P$-value $\leq 0.01)$ pairwise comparison was used to assess the significance of the differences found between the 'HRE' genotypes and the wild type (Col-0) under hypoxic conditions at the time points considered. The asterisk $(*)$ Indicates an ethanol production significantly from the wild type at the time point examined.

time points during the course of $8 \mathrm{~h}$ of hypoxia (Figure 6). Real-time qPCR analysis showed that all four genes were significantly up-regulated in the 35S::HRE 1 plants compared with the wild type. Interestingly, HRE1 over-expression also positively affects the expression of HRE2 under hypoxia. Apparently, over-expression of HRE1 only enhanced the effect of the hypoxic treatment, since it did not significantly alter the expression of SUS1, SUS4 and HB1 under aerobic conditions. Only $A D H$ expression was higher in normoxic 35S::HRE1 plants compared with the wild type (Col-0), although to only one-tenth of the levels induced by low oxygen. No significant differences were observed in the 35S::HRE2 plants. Null mutants of HRE1 and HRE2 ( hre 1 and hre2) were not impaired in the expression of the four markers, but the double mutant hre1hre2 showed a drastic decrease in the expression of anaerobic genes $4 \mathrm{~h}$ after the onset of hypoxia (Figure 6).

To identify DNA sequences that act as candidate ciselements bound by HRE1, we focused on the 22 genes that showed a significantly higher hypoxic increase in 35S::HRE1

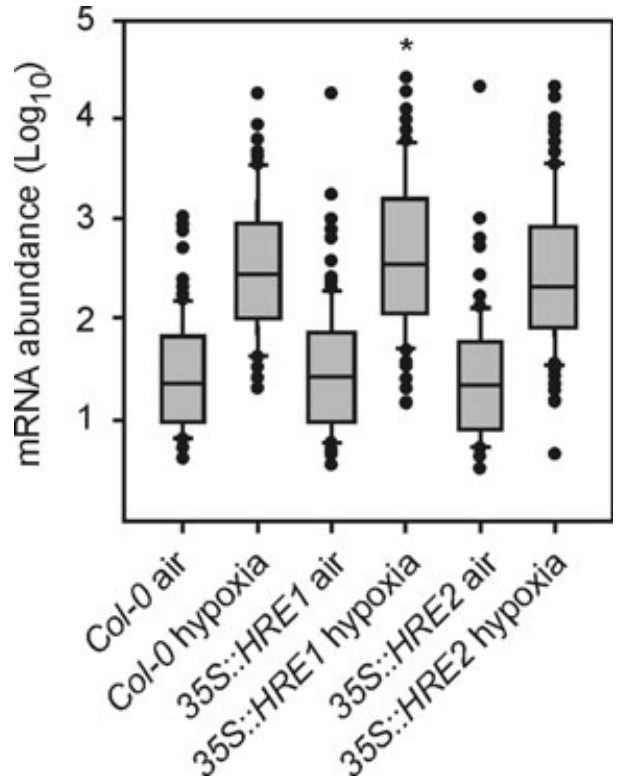

Figure 5. Effects of HRE1 and HRE2 over-expression on anaerobic genes. The box plot shows the overall effects of hypoxia on the expression of the anaerobic cluster (Table S5) in the different genotypes. $P$-value $\leq 0.01$.

compared with the wild type (Col-0) (Table S2). An analysis of the region upstream of the start of transcription (up to 1000 bp), using Toucan software (Stein et al., 2003, 2005), revealed no significant $(P \leq 0.01)$ over-representation of any known cis-element bound by transcription factors, including the GCC box which represents the putative ERF-binding site (Wang et al., 2009). On the other hand, a search for new motifs led to the identification of $A$ - (or $\mathrm{T}$-) rich regions that might represent a structural requirement for the binding of the HREs. Interestingly, A- and T-rich motifs have already been reported to be present in the promoters of low-oxygen responsive genes in several species (Mohanty et al., 2005).

\section{HRE1 and HRE2 are regulated at transcriptional and post-transcriptional levels, respectively}

The very first events in the molecular responses to environmental stimuli are likely to be independent of gene transcription or protein synthesis since the regulatory proteins involved should be constitutively present in the cell in an inactive form and quickly activated by post-translational modifications to allow a fast response. Since HRE1 and HRE2 are already induced after $30 \mathrm{~min}$ of imposition of hypoxia (van Dongen et al., 2009), we tested whether they require newly synthesized proteins to be up-regulated. A previous microarray analysis of transcriptional changes in response to cycloheximide ( $\mathrm{CHX}$ ), an inhibitor of the ribosome release from mRNA, showed that $\mathrm{CHX}$ treatments increase mRNA levels for HRE2 (NASCArrays-189). We used CHX and emetine (EMT), two different inhibitors of protein synthesis, to test whether these two compounds affect induction of HRE1 

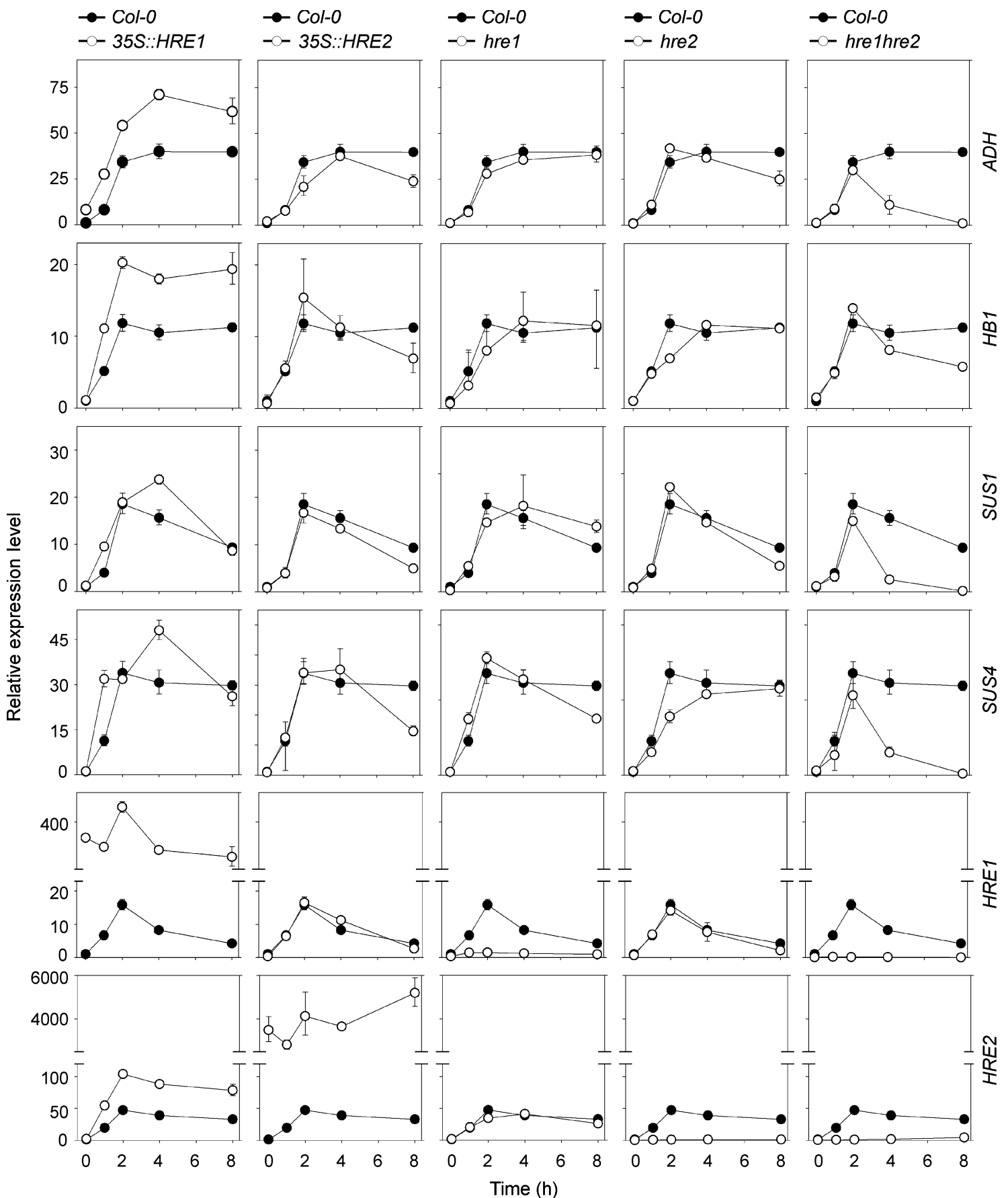

Figure 6. Effects of HRE1 and HRE2 on the expression of anaerobic genes.

The expression of four anaerobic genes ( $A D H, H B 1$, SUS1 and SUS4) and of HRE1 and HRE2 was analysed under hypoxia (1\% $\mathrm{O}_{2}$ ) in 7-day-old Arabidopsis seedlings. Five genotypes were used: Col-0 (wild type), 35S::HRE1, 35S::HRE2, hre1, hre2 and hre1hre2. The mRNA level in whole seedlings was measured by real-time quantitative PCR. Relative expression levels are shown as fold change values ( $1=$ Col-0, time 0$)$. Data are mean $\pm \operatorname{SD}(n=3)$. In each individual graph, empty circles represent the transgenic or mutant genotypes (as indicated in the legend) and the filled circles identify the wild-type (Col-0) plants.

and HRE2 under hypoxia. Cycloheximide and EMT prevented the induction of both $A D H$ (used as a control) and HRE1 under low oxygen in Arabidopsis seedlings, but not HRE2, whose expression was increased to a higher extent in aerobic conditions (Figure 7a). This suggests that the induction of $H R E 1$ and $A D H$ requires the translation of one or more transcription factors, which should take place during the first 30 min after stress imposition. Dolferus et al. (1994) identified MYB2 (At2g47190) as a transcription factor that plays a major role in the induction of $A D H$ and other genes 

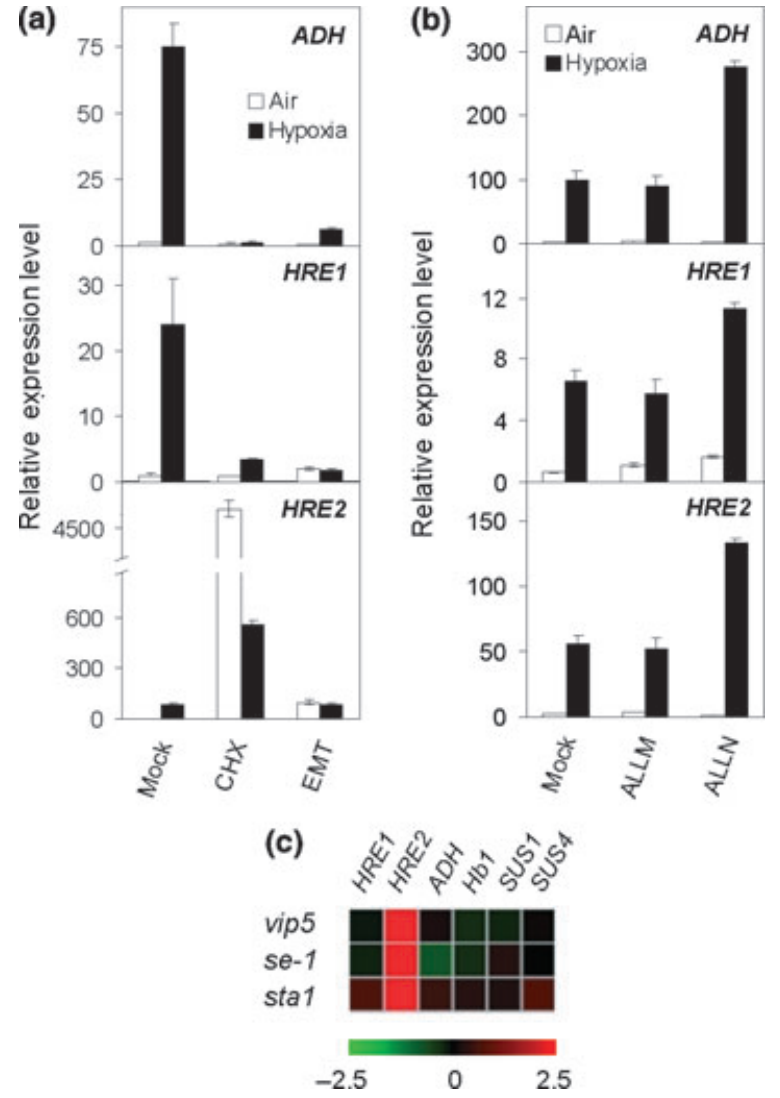

Figure 7. Regulation of HRE1 and HRE2 expression.

(a) Effects of protein synthesis inhibitors. Expression of HRE1, HRE2 and ADH genes in the absence and presence of $10 \mu \mathrm{M}$ emetine (EMT) or $50 \mu \mathrm{M}$ cycloheximide $(\mathrm{CHX})$, under aerobic and hypoxic conditions. The EMT and $\mathrm{CHX}$ were added $1 \mathrm{~h}$ before the beginning of the hypoxic treatment $\left(1 \% \mathrm{O}_{2}\right.$, $4 \mathrm{~h})$. The mRNA level in whole seedlings was measured by real-time quantitative PCR. Relative expression levels are shown as fold change values ( 1 = mock air). Data are mean $\pm \operatorname{SD}(n=3)$.

(b) Effects of proteasome inhibitors. Expression of HRE1, HRE2 and ADH genes in the absence (mock) and presence of $100 \mu \mathrm{M} \mathrm{N}$-acetyl-Leu-Leumethyoninal (ALLM) or $100 \mu \mathrm{m} N$-acetyl-Leu-Leu-norleucinal (ALLN) under aerobic (empty bars) and hypoxic $\left(1 \% \mathrm{O}_{2}, 2 \mathrm{~h}\right)$ conditions (filled bars). Ethanol $10 \mathrm{~mm}$ was used as a mock control. The mRNA level in whole seedlings was measured by real-time quantitative PCR. Relative expression levels are shown as fold change values $(1=$ mock, time 0$)$. Data are mean $\pm \operatorname{SD}(n=3)$.

(c) Effects of mRNA-processing and micro (mi)RNA-small interfering (si)RNA production mutants on the expression of HRE2. The Genevestigator heat map (Hruz et al., 2008) shows the expression values of HRE1, HRE2 and four anaerobic genes (ADH, HB1, SUS1 and SUS4) in Arabidopsis mutants involved in mRNA processing and miRNA-siRNA production under aerobic conditions: vip5 (vernalization independent 5), se-1 (serrate-1), sta1-1 (stabilized1-1). Microarray data have been retrieved from the ArrayExpress (vip5, E-MEXP-838) and GEO (se-1, GDS1047, sta1-1, GDS2102) databases.

upon anaerobiosis. MYB2 binds the $\mathrm{GC}$ box present in the promoter region of $A D H$ (Hoeren et al., 1998). A reduction in MYB2 expression in the myb2 mutant, however, did not affect the expression of either HRE1 or HRE2 (Figure S2).

The observed induction of HRE2 by $\mathrm{CHX}$ and EMT (Figure 7a) led us to speculate about the existence of a negative regulatory mechanism acting in the air which is suppressed by low oxygen. Treatment with $10 \mu \mathrm{M}$ MG132, an inhibitor of the 26S proteasome, was shown to increase the mRNA levels of HRE2 (NASCArrays-190). Two different proteasome inhibitors [ $N$-acetyl-Leu-Leu-norleucinal (ALLN) and $\mathrm{N}$-acetyl-Leu-Leu-methyoninal (ALLM)] did not affect or even increase HRE2 expression under anaerobic conditions (Figure 7b). We therefore ruled out the involvement of the proteasome in the hypoxic regulation of HRE2. Three mutants for the genes involved in mRNA processing, vip5, sta1-1 and se1, showed higher expression of HRE2 in air (Figure 7c), suggesting that HRE2 is regulated post-transcriptionally at the level of mRNA stability. According to this model, HRE2 is constitutively transcribed at a high level but its mRNA is highly unstable under normoxia, while under anaerobic conditions it suddenly becomes stable.

\section{DISCUSSION}

The ERF family encodes for proteins that belong to the AP2 superfamily, characterized by a single ERF DNA-binding domain (Nakano et al., 2006). Previous characterization of orthologs in several species, including hot pepper (Capsicum annum), tobacco (Nicotiana tabacum), pepper (Piper nigrum) and wheat (Triticum aestivum), described these proteins as nuclear-localized which were able to positively regulate gene transcription because of a single or multiple activation domains (Lee et al., 2004, 2005; Xu et al., 2007). Both HRE1 and HRE2 are indeed localized in the nucleus (Figure $2 \mathrm{~d}$ ). There are five members of ERF group VII in the Arabidopsis genome: At1g53910 (RAP2.12), At1g72360 (HRE1), At2g47520 (HRE2), At3g14230 (RAP2.2) and $A t 3 g 16770$ (RAP2.3, also known as AtEREBP). In dicots, such as poplar (Zhuang et al., 2008) and grape (FL and PP, unpublished), only a few members of this ERF group were identified, while in grasses the number of ERF group VII members is at least twice (rice) that in dicots or even more (maize) (Figure 1b). Rice Sub1A, an ethylene and flooding inducible group VII ERF, strongly affects submergence tolerance in rice (Xu et al., 2006). Among the effects of Sub1A, it is worth highlighting its role as an enhancer of fermentative metabolism, mostly through the induction of $A D H$ (Fukao et al., 2006). Sub1A, however, also acts as a repressor of the genes involved in carbohydrate mobilization through sucrose synthase (Fukao et al., 2006) and in gibberellinmediated adaptation, such as cell expansion (Fukao and Bailey-Serres, 2008).

We identified two hypoxia-inducible group VII ERFs in Arabidopsis (named HRE1 and HRE2) whose regulation and effects during anaerobiosis partly resemble those of Sub1A. Unlike Sub1A and Sub1C, HRE1 and HRE2 are not induced by ethylene (NASCARRAYS-32) but respond rapidly to oxygen deprivation (van Dongen et al., 2009). Induction of HRE 1 and HRE2 genes under hypoxia is very likely to result in an increase of the respective protein levels, since their mRNAs have been shown to be associated with polyribosomes under conditions of oxygen deprivation 
(Branco-Price et al., 2005, 2008). Both genes appear to be required for a prolonged anaerobic response, thus maintaining the expression of some anaerobic genes under low oxygen. Indeed, the double mutant hre1hre2 displayed a normal induction of anaerobic genes but failed to maintain the high expression $4 \mathrm{~h}$ after the onset of hypoxia (Figure 6). When over-expressed, only HRE1 was able to increase the hypoxic induction of the anaerobic genes, whereas 35S::HRE2 does not show any difference compared with the wild type. Moreover, expression of the $A D H$ gene is higher under aerobic conditions in 35S::HRE1 plants, compared with the wild type. RAP2.12, another member of ERF group VII, has been reported to induce $A D H$ through the interaction with its promoter (Papdi et al., 2008). However, it is unclear whether the induction triggered by RAP2.12 overexpression is comparable with that caused by hypoxia. Remarkably, over-expression of the most similar paralogue of $R A P 2.12, R A P 2.2$, did not affect the expression of $A D H$ or any other anaerobic gene (Welsch et al., 2007) in air. RAP2.2 and RAP2.12 were not up-regulated in hypoxic Arabidopsis seedlings (Figure 2a). In addition to ADH, SUS1 and SUS4 were also up-regulated in 35S::HRE1 (Figure 6), suggesting that the analogies with $S u b 1 A$, a repressor of sucrose synthase (Fukao et al., 2006), are limited.

It is thus unlikely that HREs play a role in carbohydrate conservation, suggesting different survival strategies in rice and Arabidopsis. Indeed, tolerance to anoxia is enhanced in 35S::HRE 1 plants and reduced in hre 1 hre 2 plants. The lower expression of the genes involved in lipid modifications associated with oxidative stress tolerance in 35S::HRE1 plants (Table S2) suggests that in these plants the consequences of hypoxia are attenuated, thus preserving membrane integrity and resulting in a reduced need for ROS scavenging and protective enzymes. Lipid peroxidation and membrane damage are associated with flooding and anoxic stress, although it is not clear whether this is mainly a consequence or a cause of cell death under oxygen deprivation (Licausi and Perata, 2009).

$H R E 1$ appears to act as an enhancer rather than a trigger of the anaerobic response(s), since the induction of $A D H$ and other anaerobic genes is preserved in the hre 1 mutant. Although HRE2 did not appear to trigger the same effects as HRE1, only the double mutant hre $1 \mathrm{hre} 2$ displayed a reduced (but not abolished) anaerobic response (Figure 6). This suggests that these two ERF proteins have a partially redundant molecular function, although HRE2 alone was not able to up-regulate the anaerobic genes (Figure 6). The hypoxic induction patterns of HRE1 and HRE2 were different (Figure 2), suggesting that their hypoxic regulation relies on different mechanisms. HRE1 was only transiently induced with a peak 2-4 $\mathrm{h}$ after the onset of hypoxia (Figure 2a), while HRE2 was rapidly induced and its mRNA steady state was high throughout the $(8 \mathrm{~h})$ duration of the stress (Figure 2a). Furthermore, while HRE2 did not require de novo protein synthesis to be up-regulated, HRE1 did. In fact, the translation inhibitors EMT and $\mathrm{CHX}$ prevented the expression of many anaerobic genes such as $A D H$ and $H R E 1$ but not HRE2, which is instead induced by the addition of both inhibitors, even in aerobic conditions (Figure 7a).

It is tempting to speculate that HRE2 is repressed under aerobic conditions by a labile negative regulator which, under hypoxic conditions, is not synthesized. This would fit nicely with the observation of a general repression of translation under low-oxygen conditions aimed at saving energy (Branco-Price et al., 2008). However, proteasome inhibitors could not prevent induction of HRE2 under low oxygen (Figure 7b). It therefore seems more likely that regulation of this gene occurs at the mRNA level. Indeed mutants impaired in mRNA maturation and micro (mi)RNA production, such as vip5, se and sta1-1, accumulated HRE2 in air but not other typical hypoxic genes. This thus indicates that secondary effects on plant morphology caused by the mutation do not affect the availability of oxygen for the cell (Figure 7c). VIP5 (Vernalization Independent 5) encodes for the orthologue of yeast RTF1, a protein that plays a role in post-transcriptional events acting on mRNA stability (Mueller et al., 2004; Oh et al., 2004). STA1 (Stabilized1) is part of a nuclear surveillance system that selectively degrades specific, dangerous, mRNAs before they exit the nucleus (Lee et al., 2006). Serrate1 (SE-1) encodes for a zincfinger protein involved in pre-mRNA splicing and premiRNA processing (Yang et al., 2006). All this evidence suggests that HRE2 is constitutively transcribed but its mRNA is degraded in air. No other mutant listed in Genevestigator (Hruz et al., 2008) and involved in the biogenesis or action of miRNAs or trans-acting small interfering (si)RNAs exhibited a significant difference in HRE2 mRNA (data not shown). The positive effect of $\mathrm{CHX}$ and EMT on HRE2 could then be explained by the stabilizing effect of these substances on mRNA, since protein synthesis inhibitors interfere with specific mRNA decay mechanisms (Lopez et al., 1998). Assuming that HRE2 mRNA is unstable in air, this should reside in its non-translated regions. This is because HRE2 is accumulated at high levels in transgenic plants that over-express its coding sequence devoid of untranslated regions. HRE1 might be involved in the upregulation of $H R E 2$, since the hypoxic induction of $H R E 2$ is higher in 35S::HRE1 plants.

A possible explanation for the different effects of HRE1 and HRE2 lies in an analysis of their protein structure (Figure 1a). The other group VII ERFs RAP2.2, RAP2.3 and RAP2.12 act as positive regulators of gene transcription (Buettner and Singh, 1997; Welsch et al., 2007; Papdi et al., 2008) and RAP2.2 possesses a DNA-binding domain followed by an activation domain (Welsch et al., 2007). HRE2 is shorter than the other members [171 amino acids (aa) compared with an average length of the group of 284 aa] since it lacks a large part of the region located downstream 
of the DNA-binding domain (Figure 1a). It is tempting to speculate that HRE2 lost the activating domain and requires an interaction with a partner with a trans-activating activity. Putative interactors could be selected from the large number of proteins up-regulated by low oxygen and whose function has not yet been described or to which gene transcription regulation is putatively attributed. Work is in progress to identify the possible partners of HRE2.

Under aerobic conditions, the effect of HRE1 on the expression of anaerobic genes is negligible, apart from a higher $A D H$ mRNA level in the aerobic 35S::HRE1 plants. It is thus likely that HRE1 also requires interaction with one or more proteins, either to form a complex or to be activated. Since 35S::HRE1 displays an enhanced expression of several anaerobic genes under hypoxia (Figure 6), its putative partner could also be more expressed under oxygen deprivation, thus enhancing the response as a positive loop. It is unlikely that HRE1 and HRE2 might interact since they share a conserved DNA-binding domain and have been reported to bind to DNA as monomers. On the other hand, interactions with different classes of transcription factors are highly probable. In rice, OsEBP89 interacts with OsBP5, a MYC transcription factor, to regulate the WAXY gene $(W x)$ (Zhu et al., 2003) and RAP2.3 has been reported to interact with OBF4, a protein belonging to a specific class of basic-region leucine zipper (bZIP) transcription factors (Buettner and Singh, 1997). Interestingly OsEBP89 alone binds weakly to the $W x$ promoter and requires OsBP5 to strongly activate gene transcription (Zhu et al., 2003).

In this study, we have shown that HRE1 and HRE2, two ERF transcription factors which are part of the conserved response to low oxygen in plants, are indeed involved in the regulation of the molecular response to oxygen starvation. Both HRE1 and HRE2 are required to ameliorate plant survival under anoxia. The increased tolerance in plants that over-express HRE1 is possibly due to the enhanced expression of the genes involved in the fermentative pathway. Increased ethanol production correlates positively with low-oxygen tolerance in plants over-expressing PDC (Ismond et al., 2003). HRE1 over-expression not only positively affects both $P D C$ and $A D H$ but also up-regulates ethanolic fermentation. It is tempting to propose that the enhanced tolerance of anoxia in 35S::HRE1 plants arises from a more efficient fermentative metabolism. Furthermore, the observed tolerance in 35S::HRE1 plants might be also due to the enhanced expression of a large set of anaerobic genes, including those involved in the fermentative pathway (Figure 5). Additional experiments are now required to identify the direct targets of HRE1 and HRE2. Production of plant lines expressing epitope-tagged versions of HRE1 and HRE2 coupled to chromatin immunoprecipitation (ChIP) would provide a valuable tool for the identification of target promoters and DNA motifs required for the molecular action of the two HREs. Moreover, immunopurification could also be used to identify posttranslational modifications and partners of HRE1 and HRE2 proteins. Future analyses on the post-transcriptional regulation of their molecular function, as well as the identification of putative partners, will lead to the identification of other components of the anaerobic signalosome.

\section{EXPERIMENTAL PROCEDURES}

\section{Plant material and growth conditions}

Arabidopsis thaliana, Col-0 ecotype, was used in all the experiments. Seeds were sterilized with $70 \%$ ethanol (1 min), $0.85 \%$ sodium hypochlorite (10 $\mathrm{min}$ ) and rinsed with sterile water (six times). For experiments with 4-day-old seedlings, seeds were sown in liquid Murashige-Skoog half-strength medium supplemented with $1 \%$ sucrose. Seeds were stratified for $72 \mathrm{~h}$ in the dark at $4^{\circ} \mathrm{C}$ and then transferred at $23^{\circ} \mathrm{C}$ with a 12 -h light $\left(100 \mu \mathrm{E} \mathrm{m} \mathrm{sec}^{-2}\right.$ intensity) photoperiod with shaking. To obtain 7- and 15-day-old plants, seeds were germinated on agar $(0.9 \%)$ medium supplemented with $1 \%$ sucrose using the same conditions reported above. The T-DNA insertion mutants hre1 (Salk_023445) and hre2 (Salk_052858) were obtained from the European Arabidopsis Stock Centre (NASC). Homozygous lines were identified via PCR screening of genomic DNA using gene-specific primers together with T-DNA-specific primers (Table S6). Double homozygous lines were obtained by crossing the two single mutants and then screening the $F_{2}$ generation as described above. Oxygen treatments were applied as described by van Dongen et al. (2009) and treating plants in the dark with normal air containing $21 \%$ oxygen (normoxia) or $1 \%$ oxygen (hypoxia) and $0.035 \% \mathrm{CO}_{2}$ for the time indicated in the figure legends. All treatments were started at the beginning of the light phase.

\section{Constructs and transgenic plant preparation}

Whole coding sequences of At1g72360 (HRE1) and At2g47520 (HRE2) were obtained by PCR from hypoxic cDNA using a Pfu polymerase kit (Fermentas, http://www.fermentas.com/) and cloned into pENTR-D-TOPO (Invitrogen, http://www.invitrogen.com/) to generate the entry vectors pENTR-HRE1 and pENTR-HRE2. These two entry vectors were recombined into the destination vector pK7WG2 (Karimi et al., 2002) using the LR reaction mix II (Invitrogen) to obtain the expression vectors 35S::HRE1 and 35S::HRE2.

Transgenic plants were obtained using the floral dip method (Zhang et al., 2006). T $T_{0}$ seeds were screened for kanamycin resistance and single-insertion lines were identified using real-time qPCR on genomic DNA with primer pairs annealing to the CAMV 35 S promoter, the NPTII gene and At3g10040 and At5g10040 as wild-type, single-copy genes. Homozygous $\mathrm{T}_{3}$ or subsequent generations were used in the following experiments.

To obtain GFP tagged expression of two ERF proteins, pENTRHRE1 and pENTR-HRE2, were recombined into p2FGW7 (Karimi et al., 2002). Arabidopsis mesophyll protoplasts were prepared and transformed with $10 \mu \mathrm{g}$ plasmid DNA according to Yoo et al. (2007). Fluorescence was observed with a Nikon ViCo videoconfocal microscope (http://www.nikon.com/) using a GFP filter.

\section{Real-time qRT-PCR analyses}

RNA was extracted from the seedlings, which were grown as indicated in the figure legends. Total RNA, extracted using the RNeasy kit (Qiagen, http://www.qiagen.com/) according to the manufacturer's instructions, was subjected to DNase treatment using the TURBO DNA-free kit (Ambion, http://www.ambion.com/). Five micrograms of RNA was reverse transcribed into cDNA using the 
Superscript III reverse transcriptase kit (Invitrogen). Real-time PCR amplification was carried out with the ABI Prism 7900 sequence detection system (Applied Biosystems, http://www.appliedbiosystems.com/), using a power sybr-green master mix (Applied Biosystems) and the primers described in Table S6. Ubiquitin 10 (At4G05320), PPR (AT1G62930), SAND family protein (At2g28390) and PDF2 (At1g12320), selected from the list reported in Czechowski et al. (2005), were used as housekeeping genes. Relative quantification of the expression of each individual gene was performed using the comparative threshold cycle method, as described in the ABI PRISM 7900 sequence detection system user bulletin no. 2 (Applied Biosystems).

\section{Anoxia tolerance assay}

Anoxic treatments were carried out in the dark. An enclosed anaerobic workstation (Anaerobic System model 1025; Forma Scientific, http://www.thermo.com) was used to provide an oxygenfree environment for seedling incubation. This chamber uses palladium catalyst wafers and desiccant wafers to maintain strict anaerobiosis to less than $10 \mu \mathrm{g} \mathrm{ml}^{-1} \mathrm{O}_{2}$ (according to the manufacturer's specifications). High-purity $\mathrm{N}_{2}$ was used to initially purge the chamber, and the working anaerobic gas mixture was $\mathrm{N}_{2}: \mathrm{H}_{2}$ with a ratio of 90:10. Agar plates were used to evaluate anoxia tolerance. Treatments were performed by transferring the plates containing the 7-day-old Arabidopsis seedlings to anoxia. The plates were then transferred to the light for the post-anoxic recovery $(12-\mathrm{h} / 12-\mathrm{h}$ photoperiod at $100 \mu \mathrm{E} \mathrm{m}^{-2} \mathrm{sec}^{-2}$ ). Surviving and dead plants were counted 10 days after the treatments.

\section{Metabolite and biochemistry assays}

Fourteen-day-old plants grown in vertical plates were treated in air or $1 \% \mathrm{O}_{2}$ (hypoxia) for $12 \mathrm{~h}$. Proteins were extracted from frozen root material and PDC (EC 4.1.1.1) and ADH (EC 1.1.1.1) activities were assayed as described by Ismond et al. (2003). Ethanol production by Arabidopsis seedlings was analysed enzymatically as described by Banti et al. (2008) using 7-day-old plants transferred into liquid medium and then treated in air or hypoxia $\left(1 \% \mathrm{O}_{2}\right)$ with constant shaking.

\section{Microarray analysis}

Total RNA was extracted from whole 7-day-old plants using the RNeasy kit (Qiagen). The quality of the RNA was assessed by agarose gel electrophoresis and spectrophotometry. RNA was processed for use on Affymetrix Arabidopsis Genome GeneChip arrays, as previously described (Loreti et al., 2005). Hybridization, washing, staining and scanning procedures were performed by Genopolis (University of Milano-Bicocca, http://www.genopolis.it/), as described in the Affymetrix technical manual. Microarray analysis and data quality control were performed using Robin (developed at MPI-MP Golm, http://bioinformatics.mpimp-golm.mpg.de/ projects/own/robin/). Normalization of the raw data and an estimation of signal intensities were carried out using the Genechip Robust Multichip Average (GC-RMA) methodology (Wu et al., 2004). Average expression values and their adjusted $P$-values were calculated using the Benjamini-Hochberg adjustment method (Reiner et al., 2003). Microarray datasets were deposited in a public repository with open access (accession no. GSE17099; http://www.ncbi.nlm. nih.gov/geo/).

\section{Chemical treatments}

Four-day-old seedlings were treated with $50 \mu \mathrm{M} \mathrm{CHX,} 10 \mu \mathrm{M}$ EMT, $100 \mu \mathrm{M}$ ALLM and $100 \mu \mathrm{M}$ ALLN for $1 \mathrm{~h}$ before applying the lowoxygen treatments. Distilled water was used as a mock control for the $\mathrm{CHX}$ and EMT treatments while $10 \mathrm{~mm}$ ethanol was used as a mock control for the ALLM and ALLN treatments. Plant material was sampled at the time points indicated in the figure legends.

\section{ACKNOWLEDGEMENTS}

This work was supported by Scuola Superiore Sant'Anna and the Max Planck Gesellschaft.

\section{SUPPORTING INFORMATION}

Additional Supporting Information may be found in the online version of this article:

Figure S1. Molecular and morphological phenotypes of hre1, hre2, hre1hre2, 35S::HRE1 and 35S::HRE2 genotypes.

Figure S2. Expression of HRE1 and HRE2 in the myb2 mutant.

Table S1. Genes significantly (adjusted $P$-value $\leq 0.05$ ) up- or downregulated in 35S::HRE1 plants compared with wild-type (Col-0) plants under aerobic conditions.

Table S2. Genes significantly (adjusted $P$-value $\leq 0.05$ ) up- or downregulated in 35S::HRE1 plants compared with wild-type (Col-0) plants under hypoxic $\left(1 \% \mathrm{O}_{2}, 4 \mathrm{~h}\right)$ conditions.

Table S3. Genes significantly (adjusted $P$-value $\leq 0.05$ ) up- or downregulated in 35S::HRE2 plants compared with wild-type (Col-0) plants under aerobic conditions.

Table S4. Genes significantly (adjusted $P$-value $\leq 0.05$ ) up- or downregulated in 35S::HRE2 plants compared with wild-type (Col-0) plants under hypoxic $\left(1 \% \mathrm{O}_{2}, 4 \mathrm{~h}\right)$ conditions.

Table S5. Cluster of anaerobic genes identified in the study as upregulated at least eightfold by hypoxia $\left(1 \% \mathrm{O}_{2}, 4 \mathrm{~h}\right)$ in wild-type (Col0) plants.

Table S6. List of primers used in the study.

Please note: As a service to our authors and readers, this journal provides supporting information supplied by the authors. Such materials are peer-reviewed and may be re-organized for online delivery, but are not copy-edited or typeset. Technical support issues arising from supporting information (other than missing files) should be addressed to the authors.

\section{REFERENCES}

Abe, H., Urao, T., Ito, T., Seki, M., Shinozaki, K. and Yamaguchi-Shinozaki, K. (2003) Arabidopsis AtMYC2 (bHLH) and AtMYB2 (MYB) function as transcriptional activators in abscisic acid signaling. Plant Cell, 15, 63-78.

Armstrong, W. (1979) Aeration in higher plants. Adv. Bot. Res. 7, 225-332.

Baena-González, E. and Sheen, J. (2008) Convergent energy and stress signaling. Trends Plant Sci. 13, 474-482.

Bailey-Serres, J. and Voesenek, L.A.C.J. (2008) Flooding stress: acclimations and genetic diversity. Annu. Rev. Plant Biol. 59, 313-339.

Banti, V., Loreti, E., Santaniello, A., Alpi, A. and Perata, P. (2008) Heat acclimation and cross-tolerance against anoxia in Arabidopsis. Plant Cell Environ. 31, 1029-1037.

Boutilier, K., Offringa, R., Sharma, V.K. et al. (2002) Ectopic expression of BABY BOOM triggers a conversion from vegetative to embryonic growth. Plant Cell, 14, 1737-1749.

Branco-Price, C., Kawaguchi, R., Ferreira, R.B. and Bailey-Serres, J. (2005) Genome-wide analysis of transcript abundance and translation in Arabidopsis seedlings subjected to oxygen deprivation. Ann. Bot. 96, 647660.

Branco-Price, C., Kaiser, K.A., Jang, C.J.H., Larive, C.K. and Bailey-Serres, J. (2008) Selective mRNA translation coordinates energetic and metabolic adjustments to cellular oxygen deprivation and reoxygenation in Arabidopsis thaliana. Plant J. 56, 743-755.

Buettner, M. and Singh, K.B. (1997) Arabidopsis thaliana ethylene-responsive element binding protein (AtEBP), an ethylene-inducible, GCC box DNAbinding protein interacts with an ocs element binding protein. Proc. Natl Acad. Sci. USA, 94, 5961-5966.

Christianson, J.A., Wilson, I.W., Llewellyn, D.J. and Dennis, E.S. (2009) The low-oxygen-induced NAC domain transcription factor ANAC102 affects 
viability of Arabidopsis seeds following low-oxygen treatment. Plant Physiol. 149, 1724-1738.

Czechowski, T., Stitt, M., Altmann, T., Udvardi, M.K. and Scheible, W.-R. (2005) Genome-wide identification and testing of superior reference genes for transcript normalization in Arabidopsis. Plant Physiol. 139, 5-17.

Dolferus, R., Jacobs, M., Peacock, W.J. and Dennis, E.S. (1994) Differential interactions of promoter elements in stress responses of the Arabidopsis ADH gene. Plant Physiol. 105, 1075-1087.

van Dongen, J.T., Roeb, G.W., Dautzenberg, M., Froehlich, A., Vigeolas, H., Minchin, P.E.H. and Geigenberger, P. (2004) Phloem import and storage metabolism are highly coordinated by the low oxygen concentrations within developing wheat seeds. Plant Physiol. 135, 1809-1821.

van Dongen, J.T., Froehlich, A., Ramírez-Aguilar, S.J., Schauer, N., Fernie, A.R., Erban, A., Kopka, J., Clark, J., Langer, A. and Geigenberger, P. (2009) Transcript and metabolite profiling of the adaptive response to mild decreases in oxygen concentration in the roots of Arabidopsis plants. Ann. Bot. 103, 269-280.

Elliott, R.C., Betzner, A.S., Huttner, E., Oakes, M.P., Tucker, W.Q.J., Gerentes, D., Perez, P. and Smyth, D.R. (1996) AINTEGUMENTA, an APETALA2-like gene of Arabidopsis with pleiotropic roles in ovule development and floral organ growth. Plant Cell, 8, 155-168.

Fukao, T. and Bailey-Serres, J. (2008) Submergence tolerance conferred by Sub1A is mediated by SLR1 and SLRL1 restriction of gibberellin responses in rice. Proc. Natl Acad. Sci. USA, 105, 16814-16819.

Fukao, T., Xu, K., Ronald, P.C. and Bailey-Serres, J. (2006) A variable cluster of Ethylene Response Factor-like genes regulates metabolic and developmental acclimation responses to submergence in rice. Plant Cell, 18, 20212034.

Fukao, T., Harris, T. and Bailey-Serres, J. (2009) Evolutionary analysis of the Sub1 gene cluster that confers submergence tolerance to domesticated rice. Ann. Bot. 103, 143-150.

Geigenberger, P. (2003) Response of plant metabolism to too little oxygen. Curr. Opin. Plant Biol. 6, 247-256.

Gupta, K.J., Zabalza, A. and van Dongen, J.T. (2009) Regulation of respiration when the oxygen availability changes. Physiol. Plant. 137, 383-391.

Hattori, Y., Nagai, K., Furukawa, S. et al. (2009) The ethylene response factors SNORKEL1 and SNORKEL2 allow rice to adapt to deep water. Nature, $\mathbf{4 6 0}$ 1026-1030.

Hoeren, F.U., Dolferus, R., Wu, Y., Peacock, W.J. and Dennis, E.S. (1998) Evidence for a role for AtMYB2 in the induction of the Arabidopsis Alcohol Dehydrogenase gene $(A D H 1)$ Low Oxygen. Genetics, 149, 479490.

Hruz, T., Laule, O., Szabo, G., Wessendorp, F., Bleuler, S., Oertle, L., Widmayer, P., Gruissem, W. and Zimmermann, P. (2008) Genevestigator V3: a reference expression database for the meta-analysis of transcriptomes. Adv. Bioinformatics, 2008, 420747.

Ismond, K.P., Dolferus, R., De Pauw, M., Dennis, E.S. and Good, A.G. (2003) Enhanced low oxygen survival in Arabidopsis through increased metabolic flux in the fermentative pathway. Plant Physiol. 132, 1292-1302.

Karimi, M., Inze, D. and Depicker, A. (2002) Gateway vectors for Agrobacterium-mediated plant transformation. Trends Plant Sci. 7, 193-195.

Kreuzwieser, J., Hauberg, J., Howell, K.A., Carroll, A., Rennenberg, H., Harvey Millar, A. and Whelan, J. (2009) Differential response of gray poplar leaves and roots underpins stress adaptation during hypoxia. Plant Physiol. 149 461-473.

Lasanthi-Kudahettige, R., Magneschi, L., Loreti, E., Gonzali, S., Licausi, F., Novi, G., Beretta, O., Vitulli, F., Alpi, A. and Perata, P. (2007) Transcript profiling of the anoxic rice coleoptile. Plant Physiol. 144, 218-231.

Lee, J.H., Hong, J.P., Oh, S.K., Lee, S., Choi, D. and Kim, W. (2004) The ethylene-responsive factor like protein 1 (CaERFLP1) of hot pepper (Capsicum annuum L.) interacts in vitro with both GCC and DRE/CRT sequences with different binding affinities: possible biological roles of CaERFLP1 in response to pathogen infection and high salinity conditions in transgenic tobacco plants. Plant Mol. Biol. 55, 61-81.

Lee, J.H., Kim, D.M., Lee, J.H., Bang, J.W., Kim, W.T. and Pai, H.S. (2005) Functional characterization of NtCEF1, an AP2/EREBP-type transcriptional activator highly expressed in tobacco callus. Planta, 222, 211-224.

Lee, B.-H., Kapoor, A., Zhu, J. and Zhu, J.-K. (2006) STABILIZED1, a stressupregulated nuclear protein, is required for pre-mRNA splicing, mRNA turnover, and stress tolerance in Arabidopsis. Plant Cell, 18, 1736-1749.
Licausi, F. and Perata, P. (2009) Low oxygen signaling and tolerance in plants. Adv. Bot. Res. 50, 139-198.

Liu, 0., Kasuga, M., Sakuma, Y., Abe, H., Miura, S., Yamaguchi-Shinozaki, K. and Shinozaki, K. (1998) Two transcription factors, DREB1 and DREB2, with an EREBP/AP2 DNA binding domain separate two cellular signal transduction pathways in drought- and low-temperature-responsive gene expression, respectively, in Arabidopsis. Plant Cell, 10, 1391-1406.

Lopez, P.J., Marchand, I.I., Yarchuk, O. and Dreyfus, M. (1998) Translation inhibitors stabilize Escherichia coli mRNAs independently of ribosome protection. Proc. Natl Acad. Sci. USA, 95, 6067-6072.

Loreti, E., Poggi, A., Novi, G., Alpi, A. and Perata, P. (2005) A Genome-wide analysis of the effects of sucrose on gene expression in Arabidopsis seedlings under anoxia. Plant Physiol. 137, 1130-1138.

Maeo, K., Tokuda, T., Ayame, A., Mitsui, N., Kawai, T., Tsukagoshi, H., Ishiguro, S. and Nakamura, K. (2009) An AP2-type transcription factor WRINKLED1, of Arabidopsis thaliana binds to AW-box sequence conserved among proximal upstream regions of genes Involved in fatty acid synthesis. Plant J. 60, 476-487.

Mohanty, B., Krishnan, S.P.T., Swarup, S. and Bajic, V.B. (2005) Detection and preliminary analysis of motifs in promoters of anaerobically induced genes of different plant species. Ann. Bot. 96, 669-681.

Mueller, C.L., Porter, S.E., Hoffman, M.G. and Jaehning, J.A. (2004) The Paf1 complex has functions independent of actively transcribing RNA polymerase II. Mol. Cell, 14, 447-456.

Nakano, T., Suzuki, K., Fujimura, T. and Shinshi, H. (2006) Genome-wide analysis of the ERF gene family in Arabidopsis and rice. Plant Physiol. 140 411-432.

Nicholas, K.B., Nicholas, H.B.. Jr and Deerfield, D.W. (1997) GeneDoc: analysis and visualization of genetic variation. EMBNet News, 4, 14

Ogawa, T., Pan, L., Kawai-Yamada, M., Yu, L.H., Yamamura, S., Koyama, T., Kitajima, S., Ohme-Takagi, M., Fumihiko, S. and Uchimiya, H. (2005) Functional analysis of Arabidopsis ethylene-responsive element binding protein conferring resistance to bax and abiotic stress-induced plant cell death. Plant Physiol. 138, 1436-1445.

Oh, S., Zhang, H., Ludwig, P. and van Nocker, S. (2004) A mechanism related to the yeast transcriptional regulator Paf1c is required for expression of the Arabidopsis FLC/MAF MADS box gene family. Plant Cell, 16, 2940-2953.

Ohme-Takagi, M. and Shinshi, H. (1995) Ethylene-inducible DNA binding proteins that interact with an ethylene-responsive element. Plant Cell, 7 173-182.

Pan, L., Kawai, M., Yu, L.H., Kim, K.-M., Hirata, A., Umeda, M. and Uchimiya H. (2001) The Arabidopsis thaliana ethylene-responsive element binding protein (AtEBP) can function as a dominant suppressor of Bax-induced cell death of yeast. FEBS Lett. 508, 375-378.

Papdi, C., Abraham, E., Joseph, M.P., Popescu, C., Koncz, C. and Szabados, L. (2008) Functional identification of Arabidopsis stress regulatory genes using the controlled cDNA overexpression system. Plant Physiol. 147, 528-542.

Perata, P. and Voesenek, L.A.C.J. (2007) Submergence tolerance in rice requires Sub1A, an ethylene-response-factor-like gene. Trends Plant Sci. 12, 43-46.

Reiner, A., Yekutieli, D. and Benjamini, Y. (2003) Identifying differentially expressed genes using false discovery rate controlling procedures. Bioinformatics, 19, 368-375.

Ricoult, C., Orcaray Echeverria, L., Cliquet, J.B. and Limami, A.M. (2006) Characterization of alanine aminotransferase (AlaAT) multigene family and hypoxic response in young seedlings of the model legume Medicago truncatula. J. Exp. Bot. 57, 3079-3089.

Sakuma, Y., Liu, Q., Dubouzet, J.G., Abe, H., Shinozaki, K. and YamaguchiShinozaki, K. (2002) DNA-binding specificity of the ERF/AP2 domain of Arabidopsis DREBs, transcription factors involved in dehydration- and cold-inducible gene expression. Biochem. Biophys. Res. Commun. 290, 998-1009.

Sasaki, K., Mitsuhara, I., Seo, S., Ito, H., Matsui, H. and Ohashi, Y. (2007) Two novel AP2/ERF domain proteins interact with cis-element VWRE for wound induced expression of the Tobacco tpoxN1 gene. Plant J. 50, 1079-1092.

Stein, A., Thijs, G., Coessens, B., Staes, M., Moreau, Y. and De Moor, B. (2003) TOUCAN: deciphering the cis-regulatory logic of coregulated genes. Nucl. Acids. Res. 31, 1753-1764.

Stein, A., Van Loo, P., Thijs, G., Mayer, H., de Martin, R., Moreau, Y. and De Moor, B. (2005) TOUCAN 2: the all-inclusive open source workbench for regulatory sequence analysis. Nucl. Acids. Res. 33, 393-396. 
Stockinger, E.J., Gilmour, S.J. and Thomashow, M.F. (1997) Arabidopsis thaliana CBF1 encodes an AP2 domain-containing transcriptional activator that binds to the C-repeat/DRE, a cis-acting DNA regulatory element that stimulates transcription in response to low temperature and water deficit. Proc. Natl Acad. Sci. USA, 94, 1035-1040.

Stoimenova, M., Igamberdiev, A.U., Gupta, K.J. and Hill, R.D. (2007) Nitritedriven anaerobic ATP synthesis in barley and rice root mitochondria Planta, 226, 465-474.

Tamura, K., Dudley, J., Nei, M. and Kumar, S. (2007) MEGA4: molecular evolutionary genetics analysis (MEGA) software version 4.0. $\mathrm{Mol}$. Biol. Evol. 24, 1596-1599.

Voesenek, L.A.C.J. and Bailey-Serres, J. (2009) Genetics of high-rise rice. Nature, 460, 959-960.

Wang, S., Yang, S., Yin, Y., Xi, J., Li, S. and Hao, D. (2009) Molecular dynamics simulations reveal the disparity in specific recognition of GCC-box by AtERFs transcription factors super family in Arabidopsis. J. Mol. Recognit 22, 474-479.

Weiste, C., Iven, T.R., Fischer, U., Oñate-Sánchez, L. and Droege-Laser, W. (2007) In planta ORFeome analysis by large-scale over-expression of GATEWAY ${ }^{\circledR}$-compatible cDNA clones: screening of ERF transcription factors involved in abiotic stress defense. Plant J. 52, 382-390.

Welsch, R., Maass, D., Voegel, T., Della Penna, D. and Beyer, P. (2007) Transcription factor RAP2.2 and its interacting partner SINAT2: stable elements in the carotenogenesis of Arabidopsis leaves. Plant Physiol. 145 1073-1085

Wu, Z., Irizarry, R., Gentlema, R., Murillo, F.M. and Spencer, F. (2004) A modelbased background adjustment for oligonucleotide expression arrays. J. Am. Stat. Assoc. 99, 909-917.

Wu, L., Zhang, Z., Zhang, H., Wang, X.C. and Huang, R. (2008) Transcriptional modulation of ethylene response factor protein JERF3 in the oxidative stress response enhances tolerance of tobacco seedlings to salt, drought and freezing. Plant Physiol. 148, 1953-1963.
Xu, K., Xu, X., Fukao, T., Canlas, P., Maghirang-Rodriguez, R., Heuer, S. Ismail, A.M., Bailey-Serres, J., Ronald, P.C. and Mackill, D.J. (2006) Sub1A is an ethylene-response-factor-like gene that confers submergence tolerance to rice. Nature, 442, 705-708.

Xu, Z.-S., Xia, L.-O., Chen, M. et al. (2007) Isolation and molecular characterization of the Triticum aestivum $\mathrm{L}$. ethylene-responsive factor 1 (TaERF1) that increases multiple stress tolerance. Plant Mol. Biol. 65, 719-732.

Yamamoto, S., Suzuki, K. and Shinshi, H. (1999) Elicitor-responsive, ethyleneindependent activation of GCC box-mediated transcription that is regulated by both protein phosphorylation and dephosphorylation in cultured tobacco cells. Plant J. 20, 571-579.

Yang, L., Liu, Z., Lu, F., Dong, A. and Huang, H. (2006) SERRATE is a novel nuclear regulator in primary microRNA processing in Arabidopsis. Plant $\mathrm{J}$. 47, 841-850.

Yoo, S.-D., Cho, Y.-H. and Sheen, J. (2007) Arabidopsis mesophyll protoplasts: a versatile cell system for transient gene expression analysis. Nat Protoc. 2, 1565-1572.

Youm, J.W., Jeon, J.H., Choi, D., Yi, S.Y., Joung, H. and Kim, H.S. (2008) Ectopic expression of pepper CaPF1 in potato enhances multiple stresses tolerance and delays initiation of in vitro tuberization. Planta, 228, 701-708.

Zhang, X., Henriques, R., Lin, S.S., Niu, O.W. and Chua, N.H. (2006) Agro bacterium-mediated transformation of Arabidopsis thaliana using the floral dip method. Nat. Protoc. 1, 641-646.

Zhu, Y., Cai, X.-L., Wang, Z.-Y. and Hong, M.-M. (2003) An interaction between a MYC protein and an EREBP protein is involved in transcriptional regulation of the rice Wx gene. J. Biol. Chem. 278, 4780347811.

Zhuang, J., Cai, B., Peng, R.H. et al. (2008) Genome-wide analysis of the AP2/ ERF gene family in Populus trichocarpa. Biochem. Biophys. Res. Commun. 371, 468-474. 